\title{
Structural Observation and Kinetic Pathway in the Formation of Polymeric Micelles
}

\author{
Reidar Lund, ${ }^{1, *}$ Lutz Willner, ${ }^{2}$ Michael Monkenbusch, ${ }^{2}$ Pierre Panine, ${ }^{3}$ Theyencheri Narayanan, ${ }^{3}$ \\ Juan Colmenero, ${ }^{4}$ and Dieter Richter ${ }^{2}$ \\ ${ }^{1}$ Donostia International Physics Center (DIPC), Paseo Manuel de Lardizabal, 3, 20018 Donostia-San Sebastián, Spain \\ ${ }^{2}$ Institute for Solid State Research, Forschungszentrum Jülich, D-52425 Jülich, Germany \\ ${ }^{3}$ European Synchrotron Radiation Facility (ESRF), BP 220, F-38043 Grenoble Cedex, France \\ ${ }^{4}$ Centro de Física de Materiales (Centro Mixto CSIC-UPV/EHU)/Donostia International Physics Center (DIPC), \\ Paseo Manuel de Lardizabal, 3, 20018 Donostia-San Sebastián, Spain
}

(Received 3 November 2008; published 5 May 2009)

\begin{abstract}
The route by which amphiphilic molecules self-assemble into micelles is still not fully understood. In this Letter, we present direct structural information on the birth and growth of block copolymer micelles by means of synchrotron $\mathrm{x}$-ray scattering with millisecond time resolution. Using a quantitative model, we show that the self-assembly process can be viewed as a nucleation and growth type process where the elemental growth mechanism is an exchange of single molecules.
\end{abstract}

DOI: 10.1103/PhysRevLett.102.188301

PACS numbers: 82.35.Jk, 61.05.cf, 82.35.Lr, 82.70.Uv

An archetypical example and a model system for self assembly is the family of amphiphilic A-B diblock copolymers that undergo micellization in aqueous solutions [1,2]. In such systems where the interfacial energy is usually large, the structure formation may be influenced by prominent kinetic barriers leading to a variety of trapped metastable states unable to reach their global equilibrium [3-8]. Despite recent progress on manipulation of nonequilibrium structures via kinetic control, such as by sophisticated mixing protocols $[6,9,10]$, a complete understanding of the physical mechanisms of the self-assembly process is still lacking. Therefore, a more fundamental approach to control and design such nanostructures is still a very challenging task.

Concerning micellization kinetics in particular, theories predict various growth mechanisms. Some theories exclusively favor the classical Aniansson-Wall mechanism [11] - a step-by-step insertion mechanism of single molecules (unimers) [3,12-14], while others emphasize the role of fragmentation or recombination mechanisms, i.e., fusion and fission of individual micellar entities $[15,16]$. Experimentally, appropriate data are scarce to find and what do exist are indirect in the sense that the structural evolution is not probed [17-20]. This is primarily due to the lack of experimental techniques having the correct spatial and temporal resolution with the combination of a suitable well-defined model system. In addition, there is a prominent lack of detailed physical modeling of the data. Thus, so far, results remain largely inconclusive.

Here we show that the required nanoscale spatial and millisecond temporal resolution for an in situ investigation of micellization can be reached by using synchrotron x-ray scattering. The self-assembly process of a model amphiphilic block copolymer was triggered by an interfacial tension jump experiment by rapidly changing the solvent quality for one of the blocks. Using a detailed quantitative model, we further demonstrate that the kinetic pathway proceeds by unimer exchange where only single chains are added or removed at a time.

As a model system, we employ a well-defined amphiphilic poly(ethylene-alt-propylene)-poly(ethylene oxide) (PEP1-PEO20, numbers indicate the approximate molecular weight in $\mathrm{kg} / \mathrm{mole}$ ) block copolymer [21]. Extensive previous studies have shown that these asymmetric PEPPEO diblock copolymers form well-defined starlike micelles in water-dimethylformamide (DMF) solvent mixtures $[7,8,21,22]$. This solvent pair provides a strong selectivity for PEO; however, since water and DMF have very different interfacial tensions towards the insoluble PEP block ( $\gamma \approx 46$ and $8 \mathrm{mN} / \mathrm{m}$ respectively) [21], the interfacial energy can be easily tuned by varying the composition. This tuning parameter intimately controls equilibrium unimer exchange rates $[7,8]$. Very importantly, in pure DMF only single chains (unimers) are present; but as soon as some water is added, the block copolymers spontaneously aggregate into micelles. Here we exploit this phenomenon to induce spontaneous micellization by an interfacial tension jump. This is realized in a highly accurate and reproducible way by rapidly mixing a solution of PEP1-PEO20 in pure DMF with a solvent mixture of DMF and water (80:20) using a stopped flow apparatus (BioLogic, SFM 400). The experimental setup [23] was coupled to the small angle x-ray scattering (SAXS) instrument at the high brilliance beamline, ID02, at the European Synchrotron Radiation Facility (ESRF) allowing a synchronization of extremely fast mixing $(4.5 \mathrm{~ms})$ with rapid data acquisition. We chose an optimized acquisition time of $20 \mathrm{~ms}$ and a read out pause of $140 \mathrm{~ms}$ between two acquisitions and performed stroboscopic measurements.

A typical example of the time evolution of the scattering curves is presented in Fig. 1(a). The data were recorded from a solution containing a total volume fraction of $0.25 \%$ 

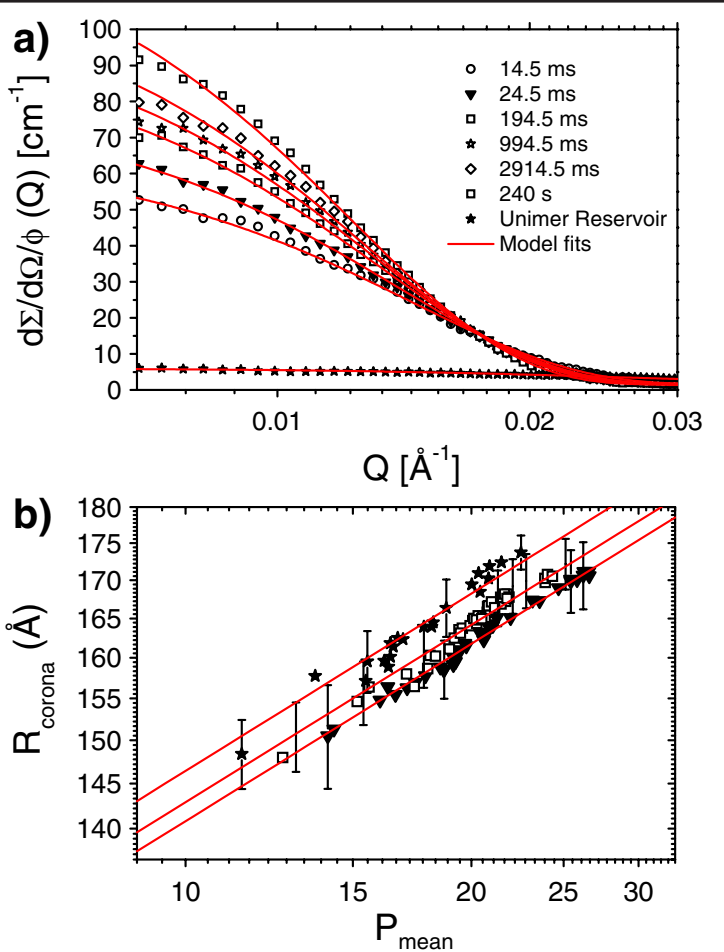

FIG. 1 (color online). (a) Normalized absolute scattering data from different times during the kinetics for the PEP-PEO system at a total volume fraction of $\phi_{\text {tot }}=0.25 \%$. Solid lines display fit results using the scattering model functions (see text for details). (b) Scaling of the coronal size, $R_{\text {corona }}$ with the aggregation number of the micelles, $P_{\text {mean }}$, both deduced from the core-shell fits, for the $0.125 \%$ (stars); $0.25 \%$ (squares), and $0.5 \%$ (triangles) solution in double logarithmical representation. The linear fits (solid lines) almost perfectly reproduce the theoretically expected scaling law for starlike micelles: $R_{\text {corona }} \sim P_{\text {mean }}^{1 / 5}$.

block copolymer in a final composition of 90:10 DMFwater (mixing equal amount of a 80:20 DMF-water solution with the PEP1-PEO20-DMF solution). The data are plotted versus the wave vector, $Q[Q=4 \pi \sin (\theta / 2) / \lambda$, where $\theta$ is the scattering angle and $\lambda$ the $\mathrm{x}$-ray wavelength], in a linear-logarithmic representation. All measurements were carried out in the very dilute regime of PEP1-PEO20 volume fractions of only $0.125 \%, 0.25 \%$, and $0.5 \%$, to minimize interparticle interferences (structure factor) facilitating a detailed structural evaluation. Even for the extremely low exposure times of $20 \mathrm{~ms}$, the high $\mathrm{x}$-ray intensity yielded very reliable and reproducible scattering signals also for very dilute solutions down to $0.125 \%$.

As exemplified in Fig. 1(a), the induced self-assembly process causes a strong increase in intensity directly reflecting the growth of the micelles in real time. In order to extract the mean aggregation number $P_{\text {mean }}$, the mean core radius, $R_{c}$, the overall micellar radius $R_{m}$, and the radial density distribution $n(r)$ accurately $(<10 \%)$ at any kinetic time, the scattering data were fitted over the whole $Q$ range with a standard core-shell model [21]. This model de- scribes the detailed structural features of the starlike micelles considering both the internal PEP core and the outer PEO shell. In order to account for some slight structure factor effects at the highest concentration, we employed Percus-Yevick structure factor which is shown to give good results for dilute block copolymer micelles [24]. Some fit results are shown in Fig. 1(a). At all times, the data are perfectly described by a spherical starlike shape in terms of the predicted radial density distribution, $n(r)$, predicted by Daoud and Cotton [25] multiplied by a cutoff function that assures a smooth decay of the density at $R_{m}: n(r) \sim$ $r^{-4 / 3} /\left(1+\exp \left[\left(r-R_{m}\right) /\left(R_{m} \sigma_{m}\right)\right]\right)$. The starlike shape is further verified by the plot in Fig. 1(b) where the average corona size, $R_{\text {corona }}=R_{m}-R_{c}$, is plotted as a function of $P_{\text {mean }}$ for all three concentrations in a double logarithmic representation. The data agree well with the prediction [25,26]: $R_{\text {corona }} \sim P_{\text {mean }}^{1 / 5}$ demonstrates consistency of the results and gives additional confidence in the scaling laws. Thus the micelles rapidly adopt a spherical starlike conformation. Finally, we attribute the very small differences $(<3 \%-4 \%)$ in the apparent size to residual structure factor effects at higher concentrations.

The concentration dependence of the micellar growth is shown in Fig. 2 in terms of $P_{\text {mean }}$ versus time for the three concentrations on a logarithmic time scale. We can fit the growth of the micelles with a phenomenological model in the form of a stretched exponential $P_{\text {mean }} \sim 1-$ $\exp \left(-(k t)^{\beta}\right)$. This yields in all cases an exponent $\beta$ of about 0.2. A trial fit to a sum of two exponentials did not give satisfactory fits, already indicating an intrinsically broad kinetic growth process. This is at odds with the existence of one or two or three distinct rate constants [18-20]. An approximate two-exponential behavior was predicted for micelle relaxation kinetics after a sudden

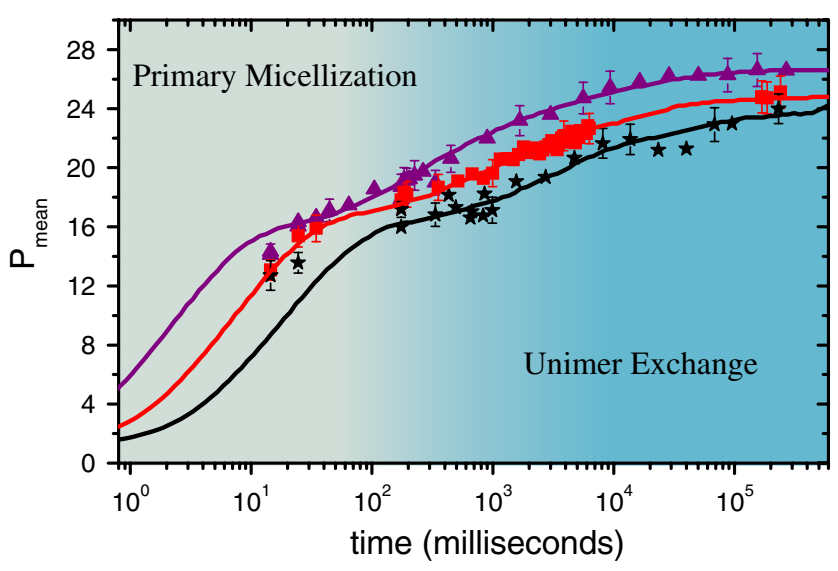

FIG. 2 (color online). Time dependence of the aggregation number, $P_{\text {mean }}$, extracted from the fits for all three total volume fractions on a logarithmical time scale; $0.125 \%$ (stars); $0.25 \%$ (squares), and $0.5 \%$ (triangles). Solid lines represent a fit using the kinetic model described in the text. The vertical lines represent typical error bars. 
external disturbance, but only very close to equilibrium $[11,13]$ that is certainly not the case here. The data at the shortest times suggest the existence of a fast initial aggregation $(t<\approx 5 \mathrm{~ms})$ that cannot entirely be resolved experimentally. This process seems to become exhausted at intermediate times leading to a "shoulder" of $P_{\text {mean }}$ that changes with concentration. The terminal relaxation towards a common equilibrium then appears to slow down with time- the overall rate increasing with concentration. The latter aspect has been observed in earlier light scattering experiments and sometimes qualitatively attributed to fusion/fission processes [20]. However, in the following we show that it can be naturally explained in terms of a simple unimer exchange model provided that the consumption of unimers is explicitly taken into account.

This model involves the simplest kinetic mechanism-a step-by-step unimer insertion or emission process:

$$
M_{P}+U \rightleftharpoons_{k_{-}}^{k_{+}} M_{P+1},
$$

where $M_{P}$ denotes the number of micelles of size $P$ and $U$ the number of unimers. The insertion and expulsion rate constants are denoted as $k_{+}=k_{+}(P)$ and $k_{-}=k_{-}(P)$, respectively.

Using this mechanism as a fundamental growth law, we construct a kinetic model for the formation of block copolymer micelles based on classical nucleation and growth theories $[14,27]$. Within the context of these theories the growth of the micelles is regulated by the micellization potential, $G(P)$-in units of $k_{B} T$-which is essentially given by the difference of the free energy of a micelle and an equivalent amount of unimers properly taking into account the translational entropy [3]:

$$
G\left(P, \phi_{1}\right)=F_{\text {micelle }}(P)-P F_{1}-(P-1) \ln \left(\phi_{1} / c_{\text {sca }}\right) .
$$

Here we have approximated the free energy by using the scaling expressions for starlike micelles $[25,26]$ : $F_{\text {micelle }}(P)=F_{\text {core }}(P)+F_{\text {corona }}(P)=\gamma^{\prime} P^{2 / 3}+\beta P^{3 / 2}$, where $\gamma^{\prime}=(36 \pi)^{1 / 3}\left(V_{\mathrm{PEP}}\right)^{2 / 3} / k_{B} T$ is proportional to the interfacial tension, $\gamma$, and the volume of the insoluble block, $V_{\mathrm{PEP}} . \beta$ is a scaling parameter close to unity that controls the entropic contribution due to the corona. $F_{1}$ is the free energy of a unimer that we here approximate using: $F_{1}=F_{\text {micelle }}(P=1) . \phi_{1}$ is the unimer volume fraction and $c_{\text {sca }}$ normalizes this experimental concentration scale to the underlying theoretical lattice model cell size. Furthermore, any correction to the assumption $F_{1}=$ $F_{\text {micelle }}(P=1)$ is included in $c_{\text {sca }}$.

Following Neu et al. [14], we assume the validity of the well-known "detailed balance" or "microscopic reversibility" principle, which gives a net creation rate in terms of the concentration, $\phi_{P+1}$, and flux, $j_{P+1}$ :

$$
\begin{aligned}
j_{P+1}= & k_{+}(P) \phi_{1}\left[\phi_{P}-\phi_{P+1} \exp \left(G\left(P+1, \phi_{1}\right)\right.\right. \\
& \left.\left.-G\left(P, \phi_{1}\right)\right)\right]
\end{aligned}
$$

which is only determined by the insertion rate constant and the potential $G\left(P, \phi_{1}\right)$ defined in thermal $k_{B} T$ units. The whole evolution of the micellar ensemble is then unambiguously given by the generic system of differential equations:

$$
\frac{\partial \phi_{P}}{\partial t}=j_{P}-j_{P+1} .
$$

Considering the very fast unimer diffusion $(\approx \mu$ s over the average unimer distance), we assume a barrier controlled insertion rate. This is proportional to the micellar cross section, $\sim P^{2 / 5}$, and the Boltzmann factor which gives $k_{+}(P)=P^{2 / 5} f \exp \left(-\left(\beta P^{1 / 2}\right)\right)$, where $f$ is the attempt frequency. The activation energy term is proportional to the derivative of the corona free energy. The stiff differential equation system that defines our kinetic model [Eq. (4)], has been solved numerically using the NAG routine D02EJF using a variable step and variable order backward differentiation method and fitted to the kinetic data (chi-square minimization).

Figure 2 shows that this theoretical model almost perfectly describes the growth behavior for the three concentrations with the same parameter set once allowing for slight variations within a few percent, reflecting stochastic minute variations in the experimental conditions. The fits yield a value of about $\gamma^{\prime} \approx 38.2$ and a spread of less than $3 \%$ This corresponds to an effective microscopic interfacial tension $\gamma \approx 19 \mathrm{mN} / \mathrm{m}$ that is of the same order as the measured macroscopic interfacial tension between PEP and this DMF-water mixture: $\gamma \approx 12 \mathrm{mN} / \mathrm{m}$ [21]. For the other parameters we obtain $\beta \approx 1.35 \pm 5 \%$, $c_{\text {scal }}=2.777 \times 10^{5}$ and $f \approx 0.034 \mathrm{~ms}^{-1}$ yielding an asymptotic value for $\phi_{1}$ of $\approx 2 \times 10^{-4}(0.02 \%)$ which is the critical micelle concentration (CMC). This low value is corroborated by the impossibility of observing free unimers at equilibrium.

The resulting time evolution of the micellar size distribution, $g(P, t)$, for $0.5 \%$, is shown in Fig. 3(a). Here we see more quantitatively what has been discussed above. The initial free unimers are consumed rapidly in a primary micellization event that resembles a classical over nucleation leading to metastable micelles (region I). These micelles can only further grow if other micelles dissolve by emitting unimers. This is particularly clear from Fig. 3(b), where we see that the micellar size, represented by $P_{\text {mean }}$, exhibits a shoulder at the time when the unimers are consumed below the CMC level (region II). Interestingly this shoulder is also characterized by a peak in the width by the Gaussian micellar distribution function. The broader distribution signifies micellar rearrangement processes where small micelles shrink and large micelles grow. At later times the distribution narrows again as the equilib- 


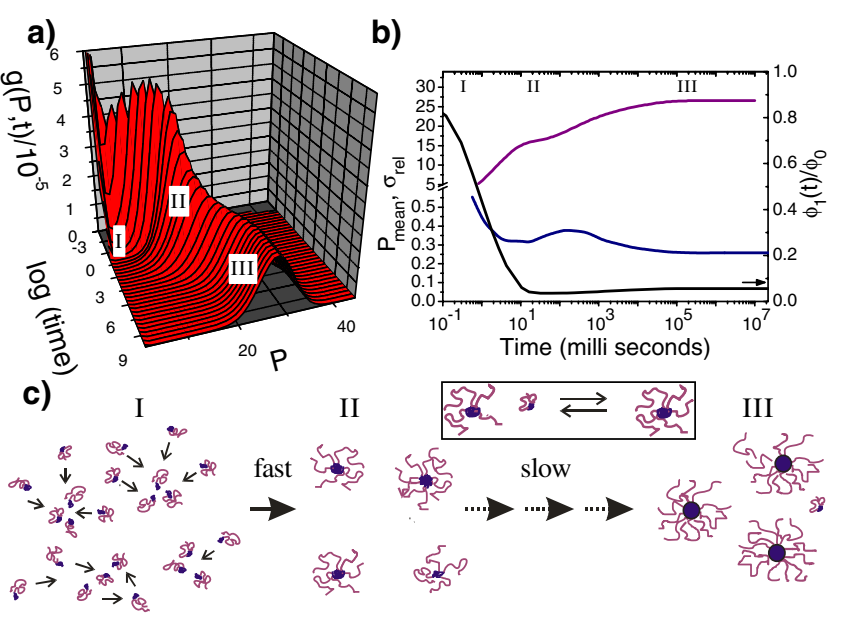

FIG. 3 (color online). (a) The time evolution of the distribution of the micellar ensemble in terms of the aggregation number, $P_{\text {mean }}$ corresponding to the fit results of the $0.5 \%$ micellar system. (b) Corresponding correlation between unimer consumption and micellar growth: time evolution of the unimer concentration (black lines) compared to the associated increase of $P_{\text {mean }}$ (purple lines). Shown is also the relative Gaussian width of the micellar distribution $\sigma_{\text {rel }}=\sigma / P_{\text {mean }}$ (blue lines). (c) Schematic view of the nucleation and growth type process of diblock copolymers leading to the final micellar structures.

rium is approached (region III). The scenario is summarized and depicted schematically in Fig. 3(c).

In summary, the kinetics of formation of block copolymer micelles have been directly observed in situ by synchrotron SAXS with millisecond resolution. Applying a quantitative model, we see that the formation and growth of micelles can accurately be described by a nucleation and growth type process governed by the elemental unimer insertion or expulsion ("Aniansson-Wall") mechanism. The kinetic pathway including the initial aggregation as well as the kinetic stability and the growth rate was shown to be intimately controlled by the system specific micellization potential $G\left(P, \phi_{1}\right)$. We anticipate that, by appropriately estimating this potential for a given system, e.g., by means of structural investigations or theoretical models, the desired properties (equilibrium or nonequilibrium) can be predicted and, in turn rationally manipulated. The design can thus be achieved not only by varying the chemical nature and sizes of the copolymer blocks, but also by much simpler physical parameters such as the polymer concentration and interfacial tension. This may prove useful to devise new predictive schemes to simple creation of pre- defined nanoparticles or techniques for controlled release of reacting agents or drugs.

The authors acknowledge the SoftComp Network of Excellence (NoE) program for support. The Basque Country Government (IT-436-07) and the Spanish Ministry of Education and Science (MAT 2007-63681) are also gratefully acknowledged. ESRF is gratefully acknowledged for the allocation of beam time.

*reidar_lund@ehu.es

[1] P. Alexandridis and B. Lindman, Amphiphilic Block Copolymers (Elsevier, Amsterdam, 2000).

[2] I. W. Hamley, The Physics of Block Copolymers (Oxford University Press, Oxford, UK, 1998).

[3] I. A. Nyrkova and A. N. Semenov, Macromol. Theory Simul. 14, 569 (2005).

[4] L. Willner et al., Europhys. Lett. 55, 667 (2001).

[5] Y.-Y. Won et al., Macromolecules 36, 953 (2003).

[6] B. K. Johnson and R. K. Prud'homme, Phys. Rev. Lett. 91, 118302 (2003).

[7] R. Lund et al., Phys. Rev. Lett. 96, 068302 (2006).

[8] R. Lund et al., Macromolecules 39, 4566 (2006).

[9] L. Zhang and A. Eisenberg, Macromolecules 32, 2239 (1999).

[10] H. Cui et al., Science 317, 647 (2007).

[11] E. A. G. Aniansson and S. N. Wall, J. Chem. Phys. 78, 1024 (1974).

[12] X. He and F. Schmid, Phys. Rev. Lett. 100, 137802 (2008).

[13] A. Halperin and S. Alexander, Macromolecules 22, 2403 (1989).

[14] J. C. Neu, J. A. Cañizo, and L. L. Bonilla, Phys. Rev. E 66, 061406 (2002).

[15] E. E. Dormidontova, Macromolecules 32, 7630 (1999).

[16] R. Pool and P. G. Bolhuis, Phys. Rev. Lett. 97, 018302 (2006).

[17] B. Bednár et al., Makromol. Chem., Rapid Commun. 9, 785 (1988).

[18] C. Honda et al., Macromolecules 27, 7660 (1994).

[19] X. Ye et al., Langmuir 23, 10366 (2007).

[20] J. Zhang et al., J. Phys. Chem. B 111, 12111 (2007).

[21] R. Lund et al., Macromolecules 37, 9984 (2004).

[22] M. Laurati et al., Phys. Rev. Lett. 94, 195504 (2005).

[23] T. M. Weiss et al., Phys. Rev. Lett. 94, 038303 (2005).

[24] J. S. Pedersen et al., Macromolecules 36, 416 (2003).

[25] M. Daoud and J. P. Cotton, J. Phys. (Paris) 43, 531 (1982).

[26] A. Halperin, Macromolecules 20, 2943 (1987).

[27] Nucleation Theory and Applications, J. W. P. Schmelzer (Wiley-VCH Verlag $\mathrm{GmbH} \& \mathrm{Co}$, KGaA, Weinheim, 2005). 